INPLASY

PROTOCOL

To cite: Xiao et al. Systematic evaluation and Mate analysis of the treatment of allergic rhinitis with needleembedding therapy. Inplasy protocol 202180029. doi: 10.37766/inplasy2021.8.0029

Received: 09 August 2021

Published: 09 August 2021

Corresponding author: Yao Xiao

1399073323@qq.com

Author Affiliation: Jiangxi University of Chinese Medicine.

Support: Unfunded.

Review Stage at time of this submission: The review has not yet started.

Conflicts of interest:

None declared.

\section{Systematic evaluation and Mate analysis of the treatment of allergic rhinitis with needle-embedding therapy}

Xiao, Y1; Xiong, J2; Zeng, Y3; Gong, M4.

Review question / Objective: To systematically evaluate the efficacy of needle-embedding therapy of allergic rhinitis.

Condition being studied: Allergic rhinitis (AR) is an inflammatory, IgE mediated disease characterized by recurrent nasal congestion, runny nose, sneezing, and/or nasal itching. As a global public health problem, the prevalence of allergic rhinitis (AR) is still on the rise throughout the world, and it is estimated to affect up to $25 \%$ of children and $40 \%$ of adults. This has significant implications for the quality of life and economic burden on those affected, the health care system and society as a whole.

INPLASY registration number: This protocol was registered with the International Platform of Registered Systematic Review and Meta-Analysis Protocols (INPLASY) on 09 August 2021 and was last updated on 09 August 2021 (registration number INPLASY202180029).

\section{INTRODUCTION}

Review question / Objective: To systematically evaluate the efficacy of needle-embedding therapy of allergic rhinitis.

Condition being studied: Allergic rhinitis (AR) is an inflammatory, IgE mediated disease characterized by recurrent nasal congestion, runny nose, sneezing, and/or nasal itching. As a global public health problem, the prevalence of allergic rhinitis (AR) is still on the rise throughout the world, and it is estimated to affect up to $25 \%$ of children and $40 \%$ of adults. This has significant implications for the quality of life and economic burden on those 
affected, the health care system and society as a whole.

\section{METHODS}

Participant or population: Patients who have intermittent or persistent AR will be included, regardless of age, gender, race, or other demographical factors. The diagnosis of $A R$ is not universally standardised, but is predominantly made clinically. We define AR based on history and physical exam consistent with an allergic cause and one or more of the following symptoms: nasal congestion, rhinorrhoea, sneezing or nasal itch. This may be supported with a positive skin prick test or the detection of high circulating blood levels of allergen-specific IgE antibody via a radioallergosorbent (RAST) test.

Intervention: Needle-embedding therapy involves a series of procedures that involve simulating body points.

Comparator: Placebo, sham acupuncture, pharmacotherapy, other types of Chinese medicine therapies.

Study designs to be included: All relevant RCTs published in English and Chinese on needle-embedding for AR can be included. Non-RCTs, reviews, case report, experimental studies, expert experience, and duplicated publications will be excluded.

Eligibility criteria: Inclusion criteria:Study designs included case-control, crosssectional, or cohort studies.Exclusion criteria:1) For duplicate literatures, only literatures with good quality and complete information should be retained, and other literatures should be deleted.2) Unable to extract data for statistical analysis;3) Poor literature quality or statistical errors;4) review; Comments. Letters.

Information sources: We will search PubMed(MEDLINE), EMBASE, Cochrane Library, Web of Science, and National Library of China Cnki, VIP, The Chinese Academy of Biomedical Research and
Wanfang will identify all published studies, regardless of language. (Deadline: August $9,2021)$.

Main outcome(s): Symptom score will be assessed as the primary outcome. The main evaluation indicators of symptom score included nasal symptoms (sneezing, runny nose, nasal itching and nasal congestion) and ocular symptoms (eye itching, foreign body sensation, red eyes, tearing). If asthma is combined, additional asthma symptoms such as wheezing, cough, shortness of breath and chest tightness should be recorded.

Additional outcome (s): I. Rhinoconjunctivitis quality of life questionnaire (RQLQ), participants with asthma can use asthma control test (ACT) II. Medicine usage and scoring III. Nasal function examination \& nasal provocation test IV. Allergen detection (e.g., skin prick test (SPT), total serum IgE detection, serum specific IgE detection will be included) $V$. Supplementary examination (e.g., nasal secretion smear, determination of specific IgE in nasal lavage fluid) VI. Side effects \& adverse events.

Quality assessment / Risk of bias analysis: Each working group consists of three members. Two members will independently screen studies and extract relevant data according to predefined selection criteria. A third member will decide whether to include or exclude indeterminate studies and examine the data extraction table. For the selected study, the first author, publication date, title, source, region, sample size and the correlation coefficient between units and AR were extracted and entered into Microsoft Excel.

Strategy of data synthesis: Data will be analyzed using Excel and STATA13.1. When heterogeneity occurs across studies, if the $p$-value was $<0.05$, a random effect model would be selected; if not, a fixed-effects model.Summary effect sizes will be calculated as RR(or AR), with $95 \%$ credible intervals (Crls). The $Q$ and $I^{2}$ statistics will be used to test for heterogeneity between included studies.Sensitivity analysis was 
performed to investigate the influence of a single study on the overall meta-analysis estimate.

Subgroup analysis: Subgroup analyses were based on the different continent, country or district (depends on the actually extracted data).

Sensitivity analysis: To assess the influence of each individual study, leave-one-out sensitivity analysis was performed iteratively by removing one study at a time to confirm that the findings were not influenced by any single study.

Country(ies) involved: China.

Keywords: Allergic rhinitis, Humans; Rhinitis, Allergic; needle-embedding therapy.

Contributions of each author:

Author 1 - Yao Xiao - The author drafted and improved the manuscript.

Email: 1399073323@qq.com

Author 2 - Jun Xiong - Revise this protocol; search strategy.

Email: xiongjun196071@163.com

Author 3 - Yuntao Zeng - Data collection; analysis of results.

Email: 1451911996@qq.com

Author 4 - Ming Gong - Data collection.

Email: 1183268181@qq.com 